\title{
High-speed optical coherence tomography as a reliable adjuvant tool to grade ocular anterior chamber inflammation.
}

\author{
Ann O. Igbre \\ Temple University \\ Mario C. Rico \\ Temple University \\ Sunir Garg \\ Thomas Jefferson University
}

Follow this and additional works at: https://jdc.jefferson.edu/willsfp

Part of the Ophthalmology Commons

Let us know how access to this document benefits you

\section{Recommended Citation}

Igbre, Ann 0.; Rico, Mario C.; and Garg, Sunir, "High-speed optical coherence tomography as a reliable adjuvant tool to grade ocular anterior chamber inflammation." (2014). Wills Eye Hospital Papers. Paper 48.

https://jdc.jefferson.edu/willsfp/48

This Article is brought to you for free and open access by the Jefferson Digital Commons. The Jefferson Digital Commons is a service of Thomas Jefferson University's Center for Teaching and Learning (CTL). The Commons is a showcase for Jefferson books and journals, peer-reviewed scholarly publications, unique historical collections from the University archives, and teaching tools. The Jefferson Digital Commons allows researchers and interested readers anywhere in the world to learn about and keep up to date with Jefferson scholarship. This article has been accepted for inclusion in Wills Eye Hospital Papers by an authorized administrator of the Jefferson Digital Commons. For more information, please contact: JeffersonDigitalCommons@jefferson.edu. 
High-speed optical coherence tomography as a reliable adjuvant tool to grade ocular anterior chamber inflammation

Ann O. Igbre ${ }^{1}$ M.D. M.P.H, Mario C. Rico ${ }^{2}$ M.D. and Sunir J. Garg ${ }^{3}$ M.D.

${ }^{1}$ The Department of Ophthalmology, Temple University School of Medicine, 3500 North Broad Street, Philadelphia, PA 19140

${ }^{2}$ Sol Sherry Thrombosis Center, Temple University School of Medicine, 3420 North Broad

Street, Philadelphia, PA 19140

${ }^{3}$ MidAtlantic Retina, The Retina Service of Wills Eye Institute, Thomas Jefferson University, 840 Walnut Street, Suite 1020, Philadelphia, PA 19107

Abbreviated title: OCT grading anterior chamber inflammation

Correspondence and reprint requests:

Sunir J. Garg, MD, FACS

Associate Professor of Ophthalmology

MidAtlantic Retina, The Retina Service of Wills Eye Institute

Thomas Jefferson University

840 Walnut Street, Suite 1020 Philadelphia, PA 19107

W: (800) 331-6634

Fax: (215) 825-9087

www.willseye.org, www.midatlanticretina.com

sunirgarg@yahoo.com

Disclosure of funding: The J. Arch McNamara Fund for Retina Research (AI and SG) and the NIH-NHLBI, K01 HL103197 (MR) 


\section{Keywords:}

Anterior chamber

Grading anterior chamber inflammation

High-speed optical coherence tomography

Inflammation

Ocular inflammatory disease

Standardization of Uveitis Nomenclature (SUN)

\section{Summary Statement:}

In this study, automated grading of the inflammation of the anterior chamber significantly correlates to the Standardization of Uveitis Nomenclature (SUN) grading system. AS-OCT is a promising technique for grading anterior chamber inflammatory cells. 


\section{ABSTRACT}

Purpose: To evaluate high-speed anterior segment (AS) optical coherence tomography (OCT) grading of AS inflammation in patients with ocular inflammation.

Methods: A retrospective, consecutive, case series study. Patients with clinically visible anterior segment inflammation in at least one eye underwent anterior segment OCT (Visante, Zeiss Meditec, Dublin, CA) with 3 to 8 a line scans per eye, performed by a trained, masked, examiner. The images were reviewed for hyperreflective spots, noise, and artifact, and these were correlated to clinical exam.

Results: Seventy- eight eyes of 41 patients were imaged. Forty-seven eyes had AC cells on clinical exam and 68 had hyperreflective spots visible on AS-OCT. There was a significant correlation (Spearman $r=0.7274$ ) between clinical exam and Visante OCT images. Several patterns of inflammation and artifact were apparent.

Conclusions: AS-OCT is a promising technique for grading AC cells. There is significant correlation between clinical and Visante grading. 


\section{INTRODUCTION}

Grading of anterior chamber (AC) inflammation is important in order to diagnose and classify patients with ocular inflammatory disease and to follow disease progression over time. Slit-lamp biomicroscopy remains the gold standard for grading anterior chamber reaction ${ }^{1}$. Although both the presence of protein in the anterior chamber (flare) and cells are noted during examination, it is the presence of cells that primarily determines treatment response. However, slit-lamp grading of anterior chamber inflammation often can be difficult. Considerable variability in grading cells exists, and even among uveitis specialists, agreement between examiners in grading AC cells is only low to moderate ${ }^{2}$.

Given the challenges in clinical grading of AC cells, a more reliable method to evaluate AC inflammation is needed. Automated grading with the laser flare meter has been used, but it has questionable accuracy especially at low levels of inflammation ${ }^{3,4}$. Recently, anterior segment optical coherence tomography (AS-OCT) has been used to image different aspects of the anterior segment, including AC cells. This method of evaluating anterior chamber inflammation may provide a more standardized and accurate way to grade inflammation. A previous study demonstrated moderate correlation between clinical grading and AS-OCT in patients with anterior uveitis, however a number of patients in this study had corneal ulcers so the results may not be generalizable to most cases of anterior uveitis ${ }^{5}$. We sought to evaluate the role of an anterior segment OCT (Visante OCT, Zeiss Meditec, Dublin, CA) for grading AC inflammation in patients with clinically visible ocular inflammatory disease. 


\section{METHODS}

\section{Obtaining clinical data}

A retrospective review of charts of patients with non-infectious uveitis who had clinically visible uveitis with anterior chamber cells and who had AS-OCT performed at the same clinic visit from the practice of one of the authors (SG) was performed. All patients had a detailed slitlamp exam after dilation and the senior author (SG) graded the anterior chamber reaction using the Standardization of Uveitis Nomenclature (SUN) criteria ranging from 0 to 4+. Briefly, patients with less than one cell per high-powered field (HPF) received grade 0. Patients with 1-5 cells per HPF had grade 0.5, those with 6-15 cells per HPF had grade 1+, 16-25 cells per HPF had grade 2+, between 26-50 cells per HPF had grade 3+, and greater than 50 cells per HPF were considered to be $4+$. If pigment was present in the anterior chamber, every attempt was made to distinguish between WBC and pigment, and pigment was not included as cell and was not factored into clinical grading of cell. All clinical grading was performed prior to ordering the AS-OCT. Exclusion criteria included eyes with infectious uveitis, and eyes with cornea edema and/or opacity.

\section{AS-OCT imaging}

Cross sectional imaging with AS-OCT (Visante OCT, Zeiss Meditec Dublin, CA) was then used to image the anterior chamber. Between 4 and 8 high-resolution corneal cross sectional scans (approximately 10mm across) were obtained through the anterior chamber by a certified 
OCT technician/ophthalmic photographer who was masked to the underlying diagnosis and clinical inflammatory level. All patients were dilated at the time of the study. After image desaturation of 30\% (Photoshop, Adobe Systems, San Jose, CA), the hyper reflective spots seen per OCT image were counted manually in a single masked fashion by one of the investigators (SG). A minimum of four representative Visante OCT images from each eye were examined and the number of hyperreflective spots from each image were averaged together. Linear hyperreflective spots were counted as artifacts and were also recorded. Correlation between anterior chamber depth and hyper reflective spots was not performed.

\section{Statistical analysis}

Positive correlation between the SUN grading and the AS-OCT grading was considered significant when the $r$ value was 0.5 or greater with a $p$ value less than 0.05 . Since the SUN grading contained ordinal data, Spearman correlation was used to measure the association between the two grading methods. Data analyses were performed using GradPad PRISM ${ }^{\circledR} 5$ Software 2010 (GraphPad software, La Jolla CA).

\section{RESULTS}

During six months, 78 eyes of 41 patients were diagnosed with anterior segmentinvolving uveitis from a number of etiologies (Table 1). There were 12 men and 29 women in the study. The average age was 47.6 years (range: 10 to 83 years). There is no statistical difference 
between gender and age of the subjects enrolled in the study. Of the 78 eyes with uveitis, 54 eyes were phakic, 23 eyes were pseudophakic and 1 eye was aphakic.

AS-OCT grading significantly correlates with clinical grading

Seventy-eight eyes were analyzed for the correlation between AC grading level and hyperreflective spots visible on OCT imaging. On OCT readings, 9 eyes had significant hyperreflective spots (greater than three standard deviations from the mean) that were considered to be imaging artifact/imaging noise and thus were excluded from analysis, resulting in 69 eyes available for further analysis. Of these 69 eyes, $22(31.8 \%)$ had inflammatory grade 0 on slitlamp examination, 20 (28.9\%) showed 0.5+ AC cells, 13 (18.8\%) showed grade 1+ AC cells, 13 $(18.8 \%)$ showed grade $2+\mathrm{AC}$ cells, and 1 (1.4\%) showed grade $3+\mathrm{AC}$ cells. No eye in this cohort had $4+$ cells. Of the 69 patients analyzed a total of 68 eyes had hyperreflective spots on OCT. These eyes had 1 to 16 hyperreflective spots on examination. Forty-seven had AC cells on clinical exam. The Spearman correlation between the anterior cell classification and that of OCT was 0.7412 with a $95 \%$ confidence interval of 0.6172 to 0.8293 , which was statistically significant $(p<0.05)$ (Figure 1). Therefore, OCT grading and the clinical grading can be used to evaluate cell infiltration of the anterior chamber. We then evaluated if OCT will be beneficial to grade inflammation among different ocular pathologies.

\section{AS-OCT as a fine tool measure of inflammation}

When the disease states were observed individually, those eyes with HLA-B27 associated ocular inflammatory disease had more significant correlation between clinical grading and OCT compared to eyes with other etiologies (Figure 2). Although twenty-two eyes had no 
clinical inflammation, 12 of these eyes had hyperreflective spots on OCT imaging. Several patterns of inflammation and artifact were apparent. Two types of artifacts were present: 1) Linear artifacts that disappeared with retraction of the lid (and not related to the lashes) were likely secondary to the tear film; and 2) numerous hyperreflective spots that were greater than 3 SD from the mean (and excluded from analysis). These spots did not appear to correlate with pigment or any other finding on clinical exam. Additionally, 17 eyes had focal hyperreflective patches on the corneal endothelium consistent with keratic precipitates (KP) (Figure 3). Fifteen eyes had both pigment and AC cells on clinical exam. There were no distinguishing characteristics between pigment and cells on Visante OCT. The correlation analysis did not significantly change with inclusion or exclusion of eyes with clinically visible pigment independent of clinically visible cell.

\section{DISCUSSION}

In this study, we observed a positive significant correlation between clinical grading of AC cells and AC-OCT imaging. We observed that although twenty-two eyes had no clinical inflammation, 12 of these eyes had hyperreflective spots on OCT imaging. This may be an advantage to clinical grading and provide better evaluation of AC cell grading. We also observed a direct correlation between clinical grading and OCT hyper-reflective spots for eyes with HLAB27 compared to eyes with other etiologies. Although we would not expect AS-OCT to be more accurate in some disease states compared to others, this finding suggests AS-OCT used to measure anterior cell inflammation may be more accurate in certain diseases. Larger series are needed to confirm this observation. 
The use of OCT for the grading of anterior chamber inflammation has been suggested in a recent study. ${ }^{5}$ The authors also found a good correlation between clinical and AS-OCT grading. However, their study included a number of patients with corneal edema and poor anterior chamber visibility on clinical exam ${ }^{5}$. In our study, none of the patients had corneal edema. Moreover, as all patients in our study had a clear view of the anterior chamber, grading of anterior segment inflammation may have been more reliable.

The manner through which inflammatory cells are graded clinically and by OCT is important. Clinically, the number of cells in a given range placed into an ordinal scale, however in each group there are a wide range of cells. For example, using the SUN criteria, an eye with $2+$ cells could have anywhere between 16 to 25 cells, and at a clinical grade of $3+$, the range in the number of cells is even greater and ranges from 26 to 50 . In contrast, the OCT grading system is a cardinal scale in which the number of hyperreflective spots counted represents the actual number of spots. Comparison of ordinal and cardinal systems can be challenging and can skew the correlation. Further complicating this comparison is that grading of anterior chamber cell is challenging even among uveitis specialists. ${ }^{2}$ As the gold standard measurement has variability, it can make comparisons to a new grading method such as OCT more challenging, but it also highlights the need for a more standardized, and automated, grading system.

Our study is limited by its retrospective nature, and to a medium sized cohort of eyes. An inherent limitation to the AS-OCT imaging device used in this study is that it is a static, twodimensional device whereas slit-lamp grading is achieved from a kinetic three-dimensional scan. Further work using both two dimensional, as well as three-dimensional AS-OCT is needed to address this issue. Furthermore, no specific protocol existed to instruct the OCT technician to image the anterior segment at certain points, for example at $0,45,90$, and 135 degrees. We feel 
that giving the examiner, in this study the certified photographer/OCT technician, the flexibility to look for hyperreflective spots on OCT more closely mirrors clinical grading in which the physician moves the slit beam in order to achieve optimal visualization of inflammatory cells. Few patients in this series had $3+$ and $4+$ inflammation, thus we cannot comment on the utility of this technique at higher levels of inflammation. On AS-OCT we were not able to distinguish hyperreflective spots due to cells from those due to pigment and in this series it did not significantly affect correlation, possibly because pigment particles may be smaller than current OCT technology can resolve. We did not systematically compare clinical grading of flare to the AS-OCT findings. Additionally, this was a cross sectional study that did not follow patients over time and we did not compare inflammation pre- and post-dilation. Future studies could examine patients over time in order to evaluate the utility of this tool to assess treatment response.

In conclusion, automated grading of anterior segment inflammation shows significant correlation with the SUN grading system, and will hopefully reduce the variability associated with clinical grading of inflammation. Technological improvements, including three-dimensional imaging may allow more accurate cell counts. This may enable a shift away from use of an ordinal scale to a cardinal scale, which will be useful not only for assessing clinical response, but also for research protocols and therapeutic studies.

\section{ACKNOWLEDGEMENTS/ DISCLOSURES}

A. Financial Disclosures: The authors have no financial interest in any of the procedures or products mentioned in this article. 
B. Contributions of Authors: Design and conduct of the study (SG); Conduct of the study (SG); management, analysis and interpretation of the data (AI, MR, SG); preparation and review (AI, $\mathrm{SG}, \mathrm{MR})$

C. Statement about Conformity with Author Information: A retrospective IRB approval was obtained from Wills Eye Institute

D: Other Acknowledgements: The authors wish to acknowledge Julia Monsonego of the Wills Eye Diagnostic and Imaging Lab for her expertise in acquiring the images in these patients.

\section{REFERENCES}

1. Jabs DA, Nussenblatt RB, Rosenbaum JT. Standardization of uveitis nomenclature for reporting clinical data. Results of the First International Workshop. Am J Ophthalmol. 2005;140(3):509-516. Prepublished on 2005/10/01 as DOI.

2. Kempen JH, Ganesh SK, Sangwan VS, Rathinam SR. Interobserver agreement in grading activity and site of inflammation in eyes of patients with uveitis. Am J Ophthalmol. 2008;146(6):813-818 e811. Prepublished on 2008/08/09 as DOI S0002-9394(08)00440-6 [pii] 10.1016/j.ajo.2008.06.004.

3. Ladas JG, Wheeler NC, Morhun PJ, Rimmer SO, Holland GN. Laser flare-cell photometry: methodology and clinical applications. Surv Ophthalmol. 2005;50(1):27-47. Prepublished on 2004/12/29 as DOI S0039-6257(04)00159-6 [pii] 10.1016/j.survophthal.2004.10.004.

4. Tugal-Tutkun I, Cingu K, Kir N, Yeniad B, Urgancioglu M, Gul A. Use of laser flare-cell photometry to quantify intraocular inflammation in patients with Behcet uveitis. Graefes Arch Clin Exp Ophthalmol. 2008;246(8):1169-1177. Prepublished on 2008/05/28 as DOI 10.1007/s00417-008-0823-6.

5. Agarwal A, Ashokkumar D, Jacob S, Saravanan Y. High-speed optical coherence tomography for imaging anterior chamber inflammatory reaction in uveitis: clinical correlation and grading. Am J Ophthalmol. 2009;147(3):413-416 e413. Prepublished on 2008/12/05 as DOI S0002-9394(08)00751-4 [pii] 10.1016/j.ajo.2008.09.024. 


\section{FIGURE CAPTIONS}

Figure 1: Plot of SUN clinical grading versus Visante OCT for anterior chamber cells. Increasing values of SUN scores correspond to increasing values of Visante OCT grading. These results demonstrate a significant positive correlation.

Figure 2: Plot of SUN clinical grading versus Visante OCT for anterior chamber cells in patients who are HLA-B27 positive, sarcoid, patients with non-granulomatous anterior uveitis, and for patients with panuveitis. HLA-B27 shows a more positive significant correlation compared to the other disease states.

Figure 3: Representative figures of anterior segment OCT images illustrating hyperreflective spots (A), artifact and noise (B), and keratic precipitates on the corneal endothelium (C). 